apparatus, impresses on one the fact that the mammalian circulation cannot carry on unless the air in the lungs is kept at practically the same pressure as the fluid environment of the body, whether that be air at atmospheric pressure or water at a hydrostatic pressure many times as great.

When a whale starts to dive, part of its buoyancy is derived from blubber and part from the volume of air in its lungs, and assuming, as I think one must, that the increasing hydrostatic pressure of the sea water acting through the body walls automatically compresses the air in the lungs in accordance with Boyle's law, it follows that as soon as the whale has reached the small depth of 5 fathoms, the air volume, and consequently the buoyancy, due to it is halved. Mr. Gray has shown that the blubber alone is insufficient to support the animal, so that the descending whale will eventually reach a certain critical depth, probably not a great one, at which the air buoyancy is so much reduced that the density of the whole animal becomes the same as that of the sea water; and if the whale dives below this and dies, it will tend to sink still farther, as in the instances Mr. Gray has described.

In diving to 500 fathoms, the air in the lungs would be compressed to about $\frac{1}{1} \frac{1}{0 \sigma}$ part of its original volume, and the lungs must shrink to a corresponding degree : it is not surprising, therefore, that anatomists have commented on their remarkable elasticity. Hunter's words (for which I am indebted to Mr. Gray) are that they are so elastic "as to squeeze out any air that may be thrown into them and to become almost at once a solid mass having a good deal the appearance, consistence, and feel of an ox's liver." The human lungs and chest are so formed that (without distortion) they cannot hold less than about $\frac{1}{5}$ of the volume of air contained after a deep inspiration, so that naked pearl divers cannot descend much beyond twenty fathoms.

The appearance of drops of condensed water in the spout of whales in the tropics may be attributed to the rapid expansion and consequent cooling of the air in the lungs as the animal ascends from a great depth.

G. C. C. Damant.

\section{Photography of the Infra-red Solar Spectrum.}

Nearly fifty years ago, Sir William Abney (Phil. Trans., Part II., p. 653, 1880, and Part II., p. 457, 1886) photographed and measured fine detail in the solar spectrum out to $\lambda 9867$. He also recorded, with low resolving power, a few broad absorption bands of greater wave-length, but he evidently observed no individual absorption lines having wavelengths exceeding that of the line mentioned. It is remarkable that, in spite of some subsequent improvements in equipment, no one has measured lines in the solar spectrum out to the limit reached by Abney. The nearest approach of which I am aware is that of Brackett (Astrophysical Journal, 53, 121 ; 1921), who measured $\lambda 9849$ and could see a few more faint lines beyond.

With the aid of plates sensitised by neocyanin, the solar spectrum is now being examined once more, using both prisms and gratings. A filter of iodine in carbon disulphide, described long ago by Prof. Tyndall, is found to be the most efficient means of preventing fog. On the prismatic plates about a dozen lines are observed between $\lambda 10,000$ and $\lambda 10,750$, one of which is fully as conspicuous as $\mathrm{H} a$. It stands clear of the great water-vapour band $\rho$, and several photographs made at various solar altitudes and on days of very different humidity fail to show any change in its appearance. On spectrograms made with the grating, dispersion $4.7 \mathrm{~A}$. per mm., it appears as a single wide line of wave-length $10049 \cdot 8 \mathrm{~A}$. The line is clearly of solar origin. Its wave-length, width, and general appearance leave little doubt that it is really the fourth member of the Paschen series of hydrogen, the calculated wave-length of which in air is $\lambda 10049 \cdot 4$. Later members of this series fall in the water-vapour band between $\lambda 9000$ and $\lambda 9600$, so that they are very difficult to observe. In addition to the lines already mentioned, fifteen others, faint and sharp, have been measured with the grating out to $\lambda 10,220$.

In spite of this extension of Abney's limit by nearly $900 \mathrm{~A}$., my experience leads to the belief that his emulsion was far more sensitive in this spectral region than any of those now available. He mentions Tyndall's filter, but seems actually to have used copperflashed ruby glass, transmitting the red as well as the infra-red. Fog due to false spectra and the diffusion of shorter wave-lengths must have caused his failure to observe the lines recorded here.

HAROLD D. BaBCOCK.

Mount Wilson Observatory, Pasadena, California,

Mar. 24

\section{Observed Relative Intensities of Stark Components of $\mathbf{H a}$.}

By means of wave mechanics, Schrödinger (Ann. d. Phys., 4, 80, 437; 1926) has made quantitative calculations of the intensities of Stark components in hydrogen which are commonly considered to be an improvement on the earlier estimates based on the correspondence principle (H. A. Kramers, D.K.D. Vidensk. Selsk., 8, 3, 287; 1919). That this is so in the case of $\mathrm{H} \beta$ was shown recently by the writers in a quantitative experimental investigation (NATURE, Oct. 23, 1926).

The greatest variation of the new theory from Prof. Stark's results, however, occurs in the parallel components of $\mathrm{Ha}$. There are three pairs of such components which have been photographed, and in the original experiments, as well as in the older quantum theory, the outside components were found to be the strongest. This is further supported by the recent calculations of Epstein on wave mechanics (Phys. Rev., 28, 695; 1926). In contrast to these results, Schrödinger finds the greatest intensity for the pair with intermediate displacements (Fig. 1). The

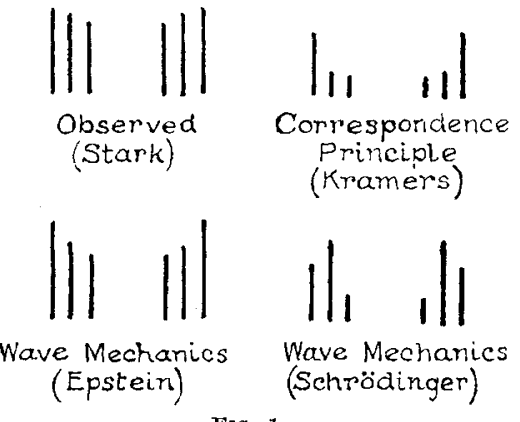

Fig. 1.

difference between Schrödinger's calculations and the observations of Stark is obviously rather large to be considered as an experimental error. Yet this is what it appears to be according to numerous plates obtained by the junior author in an extension to the earlier experiments, the new results being in general agreement with the calculations of Schrödinger.

The new photographs are taken by the Lo Surdo

No. 3056, VoL. 121] 